\title{
Combinatorial intervention of prostaglandin E2 receptor and calcium sensing receptor to attenuate breast cancer cell proliferation, migration and bone metastasis (Review)
}

\author{
JAI PARKASH $^{1}$ and KAMLESH ASOTRA ${ }^{2}$ \\ ${ }^{1}$ Robert Stempel College of Public Health and Social Work, Department of Environmental and Occupational Health, \\ Florida International University, Miami, FL 33199; ${ }^{2}$ Cardiovascular Disease and General Biomedical Sciences, \\ Tobacco-Related Disease Research Program, University of California Office of President, Oakland, CA 94612, USA
}

Received September 10, 2009; Accepted November 4, 2009

DOI: $10.3892 /$ etm_00000036

\begin{abstract}
The bone metastasis of breast cancer cells causes significant mortality among women, presumably through the enrichment of the bone microenvironment with growth factors and the stimulation of osteoclastic bone resorption, which leads to large increases in local extracellular calcium concentration, $\left[\mathrm{Ca}^{2+}\right]_{0}$. These large increases in $\left[\mathrm{Ca}^{2+}\right]_{0}$ are 'sensed' by the cancer cells as well as by osteoblasts, osteoclasts and monocytes. An extracellular calcium-sensing receptor $(\mathrm{CaR})$ enables $\left[\mathrm{Ca}^{2+}\right]_{0}$ to contribute directly to this vicious cycle by 'transactivating' an epidermal-growth factor receptor (EGFR) followed by the initiation of EGFR signaling and the up-regulation of parathyroid hormone-related peptide (PTHrP) in the breast cancer cells. Prostaglandins such as prostaglandin E2 (PGE2), which result from cyclooxygenase 2 (COX2) activity in stromal and breast cancer cells, also play an important role in the metastasis of breast cancer. The direct inhibition of the PGE2 receptor EP2 can serve as a better alternative to $\mathrm{COX} 2$ inhibition as a means for cancer prevention and treatment. Since EGFR can be 'transactivated' by both $\mathrm{CaR}$ and EP2, the resulting convergence of signaling crosstalk at the level of EGFR and PTHrP offers pharmacological interventional opportunities. We hypothesize that EP2 and CaR are coordinately involved in breast cancer cell proliferation, cell migration and bone metastasis. Our hypothesis suggests a rationale for therapeutic approaches directed against breast cancer metastasis to bone via combinatorial drug interventions of EP2 and CaR functions.
\end{abstract}

Correspondence to: Dr Jai Parkash, Robert Stempel College of Public Health and Social Work, Department of Environmental and Occupational Health, Florida International University, 11200 SW 8th Street, Room HLS-594, Miami, FL 33199, USA

E-mail: parkashj@fiu.edu

Key words: calcium-sensing receptor, bone, breast cancer, metastasis, prostaglandin E2 receptor, signaling

\section{Contents}

1. Introduction

2. Rationale and hypothesis

3. Test of hypothesis

4. Clinical relevance

\section{Introduction}

Breast cancer is the second leading cause of cancer-related death in women (1). The metastasis of breast cancer to bone that occurs in more than $80 \%$ of patients with advanced disease causes bone fractures, hypercalcemia and nerve compression (2). The osteolytic breakdown of the bone matrix by bone-resorbing osteoclasts is an important determinant of homing and metastasis of breast cancer cells to bone (2). Interactions of breast cancer cells with bone-forming osteoblasts and bone-resorbing osteoclasts may possibly result in a favorable environment for the breast cancer cells to grow rapidly $(3,4)$. However, it is still a mystery why the breast cancer cells metastasize to the bone. One possibility is that altered calcium homeostasis in breast cancer cells is a trigger for their homing to a more favorable niche, namely calciumrich bone. Do dynamic calcium fluxes caused by hyperactivity of osteoclasts and simultaneous loss of osteoblasts within bone serve as a favorable niche for the invading breast cancer cells? What does the excessive amount of calcium released during osteolysis have to do with the breast cancer cell that results in further osteolysis? To address these questions at the molecular level, one needs to consider the collective roles played by calcium sensing receptor $\mathrm{CaR}$ and the prostaglandin E2 receptor, EP2, in breast cancer cells.

\section{Rationale and hypothesis}

The breast cancer cells first detach from the primary tumor, migrate through the systemic circulation and then finally reach the bone (5). The vicious cycle that operates between breast cancer cells and bone enhances the growth of tumor upon metastasis to bone (2). The osteolytic breakdown of bone 


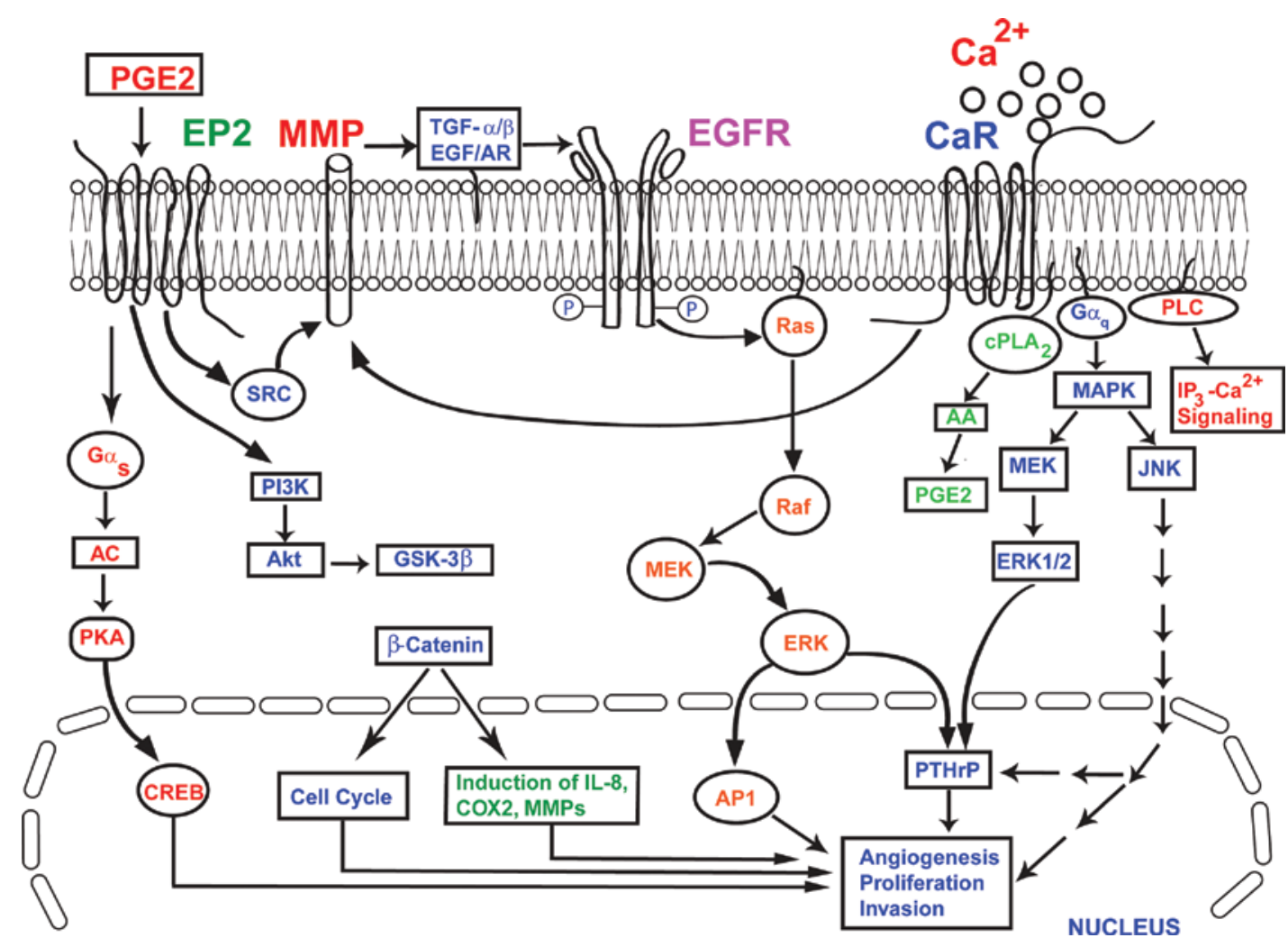

Figure 1. The convergence of EP2 and CaR signaling in breast cancer bone metastasis. The activation of $\mathrm{CaR}$ by extracellular $\mathrm{Ca}^{2+}$ leads to the activation of ERK, MAPK, cPLA2 and PLC, which in turn causes increased synthesis and secretion of PTHrP from breast cancer cells. Similarly, the 'transactivation' of EGFR by the activated CaR activates MAPK and ERK, resulting in the up-regulation of synthesis and the secretion of PTHrP from breast cancer cells. The PTHrP produced by these synergistically coupled processes in the breast cancer cells further stimulates osteoclastic bone resorption. The PGE2 activation of EP2 results in increased production of COX2, MMPs and IL-8 via the G $\alpha_{\mathrm{s}}$ and PI3K pathways, involving Akt, CREB, PKA, GSK-3 $\beta$ and $\beta$-catenin. At the same time, the activation of EP2 by PGE2 activates MMP1 and MMP2, which in turn act to release the EGFR ligands AR and TGF- $\alpha$. These then bind to EGFR and initiate EGFR signaling. In this model, the activation of EP2 and CaR, and the 'transactivation' of EGFR by both EP2 and CaR, results in increased production of PTHrP, COX2, MMPs and IL-8. This exacerbates the processes of angiogenesis, cell proliferation, invasion and the osteolytic breakdown of bone in breast cancer.

matrix releases very high concentrations of ionized calcium and phosphate from the dissolution of bone matrix (5-7). The activation of $\mathrm{CaR}$ by extracellular $\mathrm{Ca}^{2+}(8)$ can in turn 'transactivate' epidermal growth factor receptor (EGFR) and thus initiate EGFR signaling (Fig. 1) to activate mitogen-activated protein kinases (MAPK) and up-regulate synthesis and secretion of parathyroid hormone-related protein (PTHrP) from the breast cancer cells $(1,9,10)$. The PTHrP produced by the breast cancer cells further stimulates osteoclastic bone resorption.

Prostaglandin E2 (PGE2) resulting from cyclooxygenase 2 (COX2) activity has been implicated in stromal-cancer cell interactions that promote tumor growth, neovascularization and metastatic spread of many highly prevalent cancers including breast cancer (11-13). The treatment with non-steroidal antiinflammatory drugs (NSAIDs) that inhibit COX2 can reduce the risk and incidence of many types of cancer including breast cancer (13). However, in contrast to the serious potential cardiovascular complications of COX2 inhibitors, the direct inhibition of G-protein-linked prostaglandin E2 receptor (EP2) could serve as a much better alternative to COX2 inhibition as a means for breast cancer prevention and treatment $(14,15)$. EP2 couples to $G \alpha_{s}$ and stimulates cyclic AMP (cAMP) accumulation, protein kinase $\mathrm{A}$ (PKA) activation and the phosphorylation of cAMP response element binding protein (CREB), as shown in Fig. 1 (16-18). EP2 activates matrix metalloproteinases (such as MMP1 and MMP2) that in turn act to release the EGFR ligands amphiregulin (AR) and transforming growth factor $\alpha$ (TGF- $\alpha$ ), which then bind to EGFR and initiate EGFR-signaling (Fig. 1) (17-19).

$\mathrm{CaR}$ can signal through both $\mathrm{G} \alpha_{q}$ and $\mathrm{Gi}$ and activates phospholipase C (PLC), PLA2 and PLD. In breast cancer cells MCF-7 and MDA-MB-231 signaling through the CaR increases the secretion of PTHrP $(9,20)$. The PKC/MEK-1/ ERK1/2 pathway representing the CaR signaling mechanism has been proposed in the production of PTHrP from MCF-7 breast cancer cells (21). CaR activation by locally high levels of $\left[\mathrm{Ca}^{2+}\right]_{0}$ at sites of osteolytic metastases could exert proproliferative and anti-apoptotic effects and/or alter the cellular phenotype, thereby rendering the cancer cells metastasizing to the skeleton resistant to chemotherapy and/or radiation. The development of allosteric activators ('calcimimetics') and antagonists ('calcilytics') of the CaR has allowed the CaR-based therapy of the disorders of the extracellular calcium homeostasis (22).

Since EGFR can be transactivated both by $\mathrm{CaR}$ and EP2, this convergence of signaling cross-talk at the level of MMPs, EGFR and PTHrP can provide a novel opportunity to address the issues related to breast cancer proliferation and metastasis from a fresh perspective that has not been studied previously. Hence, we propose a hypothesis guiding the molecular basis for the metastatic ability of locally aggressive primary breast tumors to bone, and a rationale for combinatorial therapeutic 
interventions against metastasis. We hypothesize that 'EP2 and $\mathrm{CaR}$ are collectively involved in breast cancer cell proliferation, cell migration and bone metastasis'. As a corollary to this hypothesis, it is therefore suggested that combinatorial inhibition of EP2 and $\mathrm{CaR}$ would attenuate proliferation, migration and bone metastasis of breast cancer cells.

\section{Test of hypothesis}

To provide tests for this hypothesis, one may carry out the following studies to answer four critical questions related to the metastasis of breast cancer cells to bone and potential pharmacological interventions to prevent it:

i) Do CaR and EP2 in breast cancer cells collectively facilitate cell proliferation and inhibit apoptosis? To address this question, one can use genetic, pharmacological and immunological approaches such as a) silence the expression of EP2 and CaR to analyze the compound knockdown by breast cancer cells, b) pharmacological inhibition of EP2 using EP2 antagonists and CaR inhibition by 'calcilytics' (15,22-25), and c) inhibition of EP2 and CaR by anti-EP2 and anti-CaR antibodies. The expression of CaR and EP2 can be decreased significantly by using short hairpin RNA (shRNA) in MDA-MB-231 cells. It is also possible to generate the compound knockdown cells in which both shRNA for CaR and EP2 can be expressed in a given cell population thereby silencing both these genes and their encoded products $\mathrm{CaR}$ and EP2. The efficiency of knockdown can be determined using quantitative RT-PCR and Western blotting. In order to understand the relationship between biological functions and biochemical pathways, the loss-of-function phenotype by using RNA interference (RNAi) could be very useful. The RNAi technology has been exploited in organisms for understanding gene function via suppression of gene expression (26). The expression of a simple, 29-bp hairpin from a U6 small nucleolar RNA (snRNA) promoter can induce effective suppression of target genes when delivered either transiently or stably from integrated constructs (27). The cell proliferation and apoptosis of breast cancer cells can also be pharmacologically targeted using previously characterized doses of the anti-CaR and anti-EP2 antibodies. One may use NPS 2143 which acts as an antagonist to CaR (17) as a pharmacological agent, and use EP2 antagonist AH6809 (25). When used as single agents, these drugs should inhibit cell proliferation, inhibit the trans-endothelial migration (for the migratory capacity of MDA-MB-231 cells through an endothelial monolayer, see below), and increase the rate of apoptosis of MDA-MB-231 cells, thereby mimicking the effect of single genetic knockdown (i.e., either CaR or EP2 knockdown). However, consistent with genetic studies on combined knockdown of $\mathrm{CaR}$ and EP2, treatment with the anti-CaR and anti-EP2 combinations as well as AH6809 and NPS 2143 combination should reduce the rate of cell proliferation, reduce the trans-endothelial migration and increase the rate of apoptosis much more significantly. The rate of cell replication can be measured by the incorporation of 5-bromodeoxyuridine as defined previously $(28,29)$. In order to study changes in the rate of apoptosis, cleaved caspase-3 can be quantified by using fluorescence microscopy in combination with monoclonal antibody conjugated with fluorescein-isothiocyanate (FITC) that specifically recognizes the active form of caspase- 3 in cells.

Reduction of $\mathrm{CaR}$ or EP2 expression individually is expected to have statistically significant, yet limited, effects on cell proliferation and apoptosis. Nevertheless, silencing of $\mathrm{CaR}$ and EP2 genes is expected to increase cell death and decrease the rate of cell proliferation. By using genetic, pharmacological and immunological approaches, it is possible to determine if $\mathrm{CaR}$ and EP2 in breast cancer collectively facilitate cell proliferation and inhibit apoptosis. The pharmacological inhibition of EP2 using EP2 antagonists and CaR inhibition by 'calcilytics' can be carried out in MDA-MB-231 breast cancer cells.

It has been previously reported that when any of four lungmetastatic signature (LMS) genes is inactivated by genetic manipulation in breast cancer cells that are metastasizing to the lung, there is only a moderate inhibition of primary-tumor growth and lung metastasis (30). However, when combinations of these genes are inactivated, additive or synergistic effects are apparent, with an almost complete abrogation of both primary-tumor growth and lung metastasis when all four genes are inactivated. Gupta et al (30) also used pharmacological agents to specifically inhibit the products of the four genes. Combinatorial treatment of mice transplanted with the lung metastatic variant of the MDA-MB-231 cell line recapitulated the results of the genetic knockdown studies.

ii) Do CaR and EP2 in breast cancer collectively facilitate cell migration? The in vitro assay of trans-endothelial migration of MDA-MB-231 cells can be carried out by seeding human-umbilical vascular endothelial cells (HUVECs) into collagen-coated trans-well inserts and allowing them to grow to confluence. Breast cancer cells can then be loaded with a fluorescent cell tracker green dye before being conditioned overnight in cell culture media without growth factors. The next day, breast cancer cells can be seeded into trans-well inserts with or without a confluent HUVEC endothelial monolayer, and the wells can be fixed in $4 \%$ paraformaldehyde after $10 \mathrm{~h}$. Cells on the apical side of each insert can then be removed and the trans-well membrane mounted onto slides. The migration of MDA-MB-231 to the basolateral side of the membrane can be visualized with a fluorescence microscope. The images of 6-10 random fields across three replicate wells can be acquired for quantification, and migration of the cells is plotted as a percentage of migrating MDA-MB-231 control cells.

It is expected that trans-endothelial migration, the migratory capacity of MDA-MB-231 cells through an endothelial monolayer, will be inhibited by the a) combined knockdown of CaR and EP2, b) anti-CaR and anti-EP2 antibodies, and c) pharmacological agents NPS 2143 and AH6809. This result should provide evidence that the expression of CaR and EP2 by cancer cells can collectively promote trans-endothelial migration, a step involved in metastatic intravasation and extravasation processes.

iii) Do CaR and EP2 in breast cancer cells collectively promote bone colonization? One can utilize 2- and 3-D cultures of MC3T3-E1, a murine preosteoblast cell line as an in vitro model of bone to study the collective roles of CaR and EP2 in 
breast cancer cell colonization of the bone $(6,7,31)$. It is feasible to use both 2- and 3-D cultures (using a specially designed bioreactor) of MC3T3-E1, a murine preosteoblast cell line as an in vitro model of bone to study breast cancer colonization of bone. By adding MDA-MB-231 breast cancer cells to these 2- and 3-D cultures, one can show by using Z-stack sectioning and analysis of expression of differentiation proteins type 1 collagen, osteocalcin and osteonectin in the culture media that the combinatorial inhibition of EP2 and $\mathrm{CaR}$ by a) combined knockdown of CaR and EP2, b) antibodies against CaR and EP2, and c) pharmacological agents NPS 2143 a 'calcilytics' and AH6809 a EP2 antagonist, in breast cancer cells indeed inhibits bone colonization (7).

iv) Signaling cross-talk between EP2 and CaR at the downstream targets, MMPs and EGFR. An emerging body of evidence indicates that GPCRs are able to transactivate receptor tyrosine kinases (RTKs) including EGFR (Fig. 1) $(10,32,33)$. The initial transactivation process involves stimulation of MMPs, which results in the extracellular release of a latent membrane-spanning precursor of a member of the family of ligands known to activate these groups of receptors (19,33). These ligands, either heparin-bound (HB)-EGF or TGF- $\alpha$, then secondarily activate the EGFR to phosphorylate specific tyrosine residues residing in intracellular domains of EGFR, thereby activating downstream proteins such as MAPKs (10,34). Inhibiting MMPs by a broadly selective MMP inhibitor, GM-6001 and neutralizing heparin-bound EGF with a neutralizing antibody has been shown to prevent the CaR-mediated increases in phospho-ERK and PTHrP release, consistent with a triple-membrane-spanning signaling requirement for transactivation of the EGFR by the CaR (Fig. 1) (35). Similarly, EP2 can also promote the transactivation of EGFR expressed in cancer cells and thus initiate the EGFR-signaling network (Fig. 1) (17,18). It is possible to study convergence of signaling cross-talk both in control and in MDA-MD-231 cells a) silenced genetically by shRNA against EP2 and CaR, b) combinatorially inhibited by pharmacological agents of CaR 'calcilytics' and EP2 antagonist, and c) inhibited by antibodies directed against EP2 and CaR.

The downstream targets signaling cross-talks between EP2 and $\mathrm{CaR}$ can be determined at the level of MMPs and EGFR stimulation by both EP2 and CaR. In order to determine EGFR stimulation by EP2 and CaR, EGFR can be immunoprecipitated and probed for tyrosine phosphorylation by using polyclonal antisera against phosphotyrosine and the EGFR. The silencing of $\mathrm{CaR}$ or EP2 gene expression individually is expected to cause a statistically significant decrease in transactivation of EGFR. Nevertheless, the combinatorial silencing of $\mathrm{CaR}$ and EP2 genes is expected to significantly decrease transactivation of EGFR further. Similarly, when used as single agents, the drugs anti-CaR or anti-EP2 or AH6809 or NPS 2143 alone should inhibit transactivation of EPGR thereby mimicking the effect of single genetic knockdown (i.e., either $\mathrm{CaR}$ or EP2 knockdown). However, consistent with genetic studies on combined knockdown of $\mathrm{CaR}$ and EP2, treatment with the anti-CaR and anti-EP2 combinations as well as AH6809 and NPS 2143 combination should lead to extensive decrease in the transactivation of EGFR.
Determination of MMP1 and MMP. For analysis of MMP1 and MMP2 secreted protein, MDA-MB-231 cells can be plated in cell culture medium. The experimental conditioned media can then be collected at defined intervals and MMP1 and MMP2 concentrations can be analyzed in conditioned media using ELISA kits. The reduction of CaR or EP2 expression individually is expected to cause a statistically significant decrease in the release of MMP1 and MMP2. Nevertheless, the combinatorial silencing of $\mathrm{CaR}$ and EP2 genes is expected to decrease the release of MMP1 and MMP2 further. Similarly, when used as single agents, the drugs anti-CaR or anti-EP2 or AH6809 or NPS 2143 alone should decrease the release of MMP1 and MMP2 thereby mimicking the effect of single genetic knockdown (i.e., either CaR or EP2 knockdown). However, consistent with genetic studies on combined knockdown of CaR and EP2, treatment with the anti-CaR and anti-EP2 combinations as well as AH6809 and NPS 2143 combination should decrease the release of MMP1 and MMP2 very significantly.

\section{Clinical relevance}

The present hypothesis provides a molecular basis for the metastatic ability of aggressive primary breast tumors, and a rationale for a therapeutic approach against metastasis via combinatorial interventions involving EP2 and CaR. As we progress towards the molecular understanding of the biological functions required for metastasis, it may become possible to develop antimetastatic strategies that target metastatic cells and their interactions with newly acquired microenvironments. The inhibition of EP2 and CaR via combinatorial therapies can abate metastatic progression in a clinically relevant model of breast cancer. The inhibition of EP2 and CaR activities would benefit breast cancer patients in a number of novel ways such as a) blocking the breast cancer cell proliferation at the primary site, b) blocking the trans-endothelial migration of breast cancer cells in the vasculature, and finally c) blocking the bone breakdown. These studies can provide novel insights as to how breast cancer cells become metastatic, specifically homing to and targeting bone. This knowledge will pave the way to develop clinical strategies to prevent breast cancer metastasis to bone.

\section{Acknowledgements}

The authors acknowledge the Florida International University Foundation's Faculty Research Award to J.P., and the Research Conference Awards from the National Institutes of Health, Flight Attendant Medical Research Institute, Society for Free Radical Research International and the Oxygen Club of California to K.A.

\section{References}

1. Kingsley LA, Fournier PGJ, Chirgwin JM and Guise TA: Molecular biology of bone metastasis. Mol Cancer Ther 6: 2609-2617, 2007.

2. Kozlow W and Guise TA: Breast cancer metastasis to bone: mechanisms of osteolysis and implications for therapy. J Mammary Gland Biol Neoplasia 10: 169-180, 2005.

3. Fidler IJ: The pathogenesis of cancer metastasis. The 'seed and soil' hypothesis revisited. Nat Rev Cancer 3: 453-458, 2003. 
4. Paget S: The distribution of secondary growths in cancers of breast. Lancet 1: 571-573, 1889.

5. Kakonen SM and Mundy GR: Mechanisms of osteolytic bone metastases in breast carcinoma. Cancer 97: 834-839, 2003.

6. Doherty TM, Uzui H, Fitzpatrick LA, Tripathi PV, Dunstan CR, Asotra K and Rajavashisth TB: Mechanism of arterial calcification in the context of atherosclerosis. FASEB J 16: 577-582, 2002.

7. Doherty TM, Asotra K, Fitzpatrick LA, Dunstan CR, Qiao J-H, Wilkin DJ, Detrano RC, Shah PK and Rajavashisth TB: Calcification in atherosclerosis: bone biology and chronic inflammation at the arterial crossroads. Proc Natl Acad Sci USA 100: 11201-11206, 2003.

8. Parkash J, Chaudhry MA and Rhoten WB: Calbindin-D28k and calcium sensing receptor cooperate in MCF-7 human breast cancer cells. Int J Oncol 24: 1111-1119, 2004.

9. Sanders JL, Chattopadhyay N, Kifor O, Yamaguchi T, Butters RR and Brown EM: Extracellular calcium-sensing receptor expression and its potential role in regulating parathyroid hormone-related peptide secretion in human breast cancer cell lines. Endocrinology 141: 4357-4364, 2000.

10. Schafer B, Gschwind A and Ullrich A: Multiple G-protein receptor signals converge on the epidermal growth factor receptor to promote migration and invasion. Oncogene 23: 991-999, 2004.

11. Chang SH, Ai Y, Breyer RM, Lane TF and Hla T: The prostaglandin E2 receptor EP2 is required for cyclooxygenase 2-mediated mammary hyperplasia. Cancer Res 65: 4496-4499, 2005.

12. Hiraga T, Myoui A, Choi ME, Yoshikawa $H$ and Yoneda $T$ : Stimulation of cyclooxygenase-2 expression by bone-derived transforming growth factor- $\beta$ enhances bone metastases in breast cancer. Cancer Res 66: 2067-2073, 2006.

13. Mazhar D, Ang R and Waxman J: COX inhibitors and breast cancer. Br J Cancer 94: 346-350, 2006.

14. Bresalier RS, Sandler RS, Quan H, et al: Cardiovascular events associated with rofecoxib in a colorectal adenoma chemoprevention trial. N Engl J Med 352: 1092-1102, 2005.

15. Fulton AM, Ma X and Kundu N: Targeting prostaglandin E EP receptors to inhibit metastasis. Cancer Res 66: 9794-9797, 2006.

16. Dorsam RT and Gutkind JS: G-protein coupled receptors and cancer. Nat Rev 7: 79-94, 2007.

17. Pai R, Soreghan B, Szabo IL, Pavelka M, Baatar D and Tarnawski AS: Prostaglandin E2 transactivates EGF receptor: a novel mechanism for promoting colon cancer growth and gastrointestinal hypertrophy. Nat Med 8: 289-293, 2002.

18. Willmarth NE, Baillo A, Dziubinski ML, Wilson K, Riese DJ II and Ethier SP: Altered EGFR localization and degradation in human breast cancer cells with an amphiregulin/EGFR autocrine loop. Cell Signal 21: 212-219, 2009.

19. Doherty TM, Asotra K, Pei D, Uzui H, Wilkin DJ, Shah PK and Rajavashisth TB: Therapeutic developments in matrix metalloproteinase inhibition. Expert Opinion Therapeutic Patents 12: 665-707, 2002.

20. Brown EM and MacLeod RJ: Extracellular calcium sensing and extracellular calcium signaling. Physiol Rev 81: 239-297, 2001.

21. Lindemann RK, Braig M, Hauser CA, Nordheim A and Dittmer J Ets2 and protein kinase $\mathrm{C}$ epsilon are important regulators of parathyroid hormone-related protein expression in MCF-7 breast cancer cells. Biochem J 372: 787-797, 2003.
22. Nemeth EF, Delmar EG, Heaton WL, et al: Calcilytic compounds: potent and selective $\mathrm{Ca}^{2+}$ - receptor antagonists that stimulate secretion of parathyroid hormone. J Pharmacol Exp Ther 299: 323-331, 2001

23. Brown EM: Clinical lessons from the calcium-sensing receptor. Nat Clin Pract Endocrinol Metab 3: 122-133, 2007.

24. Mihai R, Stevens J, McKinney C and Ibrahim NB: Expression of the calcium receptor in human breast cancer - a potential new marker predicting the risk of bone metastases. Eur J Surg Oncol 32: 511-515, 2006

25. Woodward DF, Pepperl DJ, Burkey TH and Regan JW: 6-Isopropoxy-9-oxoanthene-2-carboxylic acid (AH6809), a human EP2 receptor antagonist. Biochem Pharmacol 50: 1731-1733, 1995.

26. Silva J, Chang K, Hannon GJ and Rivas FV: RNA-interferencebased functional genomics in mammalian cells: reverse genetics coming of age. Oncogene 23: 8401-8409, 2004.

27. Paddison PJ, Caudy AA, Bernstein E, Hannon GJ and Conklin DS: Short hairpin RNAs (shRNAs) induce sequencespecific silencing in mammalian cells. Genes Dev 16: 948-958, 2002.

28. Parkash J, Chaudhry MA and Rhoten WB: Tumor necrosis factor- $\alpha$ induced changes in insulin-producing $\beta$-cells. Anatom Rec A Discov Mol Cell Evol Biol 286A: 982-993, 2005.

29. Parkash J, Felty Q and Roy D: Estrogen exerts a spatial and temporal influence on reactive oxygen species generation that precedes calcium uptake in high capacity mitochondria: implications for rapid nongenomic signaling of cell growth. Biochemistry 45: 2872-2881, 2006.

30. Gupta GP, Nguyen DX, Chiang AC, et al: Mediators of vascular remodelling co-opted for sequential steps in lung metastasis. Nature 446: 765-770, 2007.

31. Dhurjati R, Krishnan V, Shuman LA, Mastro AM and Vogler EA: Metastatic breast cancer cells colonize and degrade threedimensional osteoblastic tissue in vitro. Clin Exp Metastasis 25: 741-752, 2008

32. Fisher OM, Hart S, Gshwind A and Ullrich A: EGFR signal transactivation in cancer cells. Biochem Soc Trans 31: 1203-1208, 2003.

33. Prenzel N, Zwick E, Daub H, Leserer M, Abraham R, Wallasch C and Ullrich A: EGF receptor transactivation by G-protein-coupled receptors requires metalloproteinase cleavage of proHB-EGF Nature 402: 884-888, 1999.

34. Gilmore JL, Scott JA, Bouizar Z, Robling A, Pitfield SE, Riese DJ II and Foley J: Amphiregulin-EGFR signaling regulates PTHrP gene expression in breast cancer cells. Breast Cancer Res Treat 110: 493-505, 2008.

35. MacLeod RJ, Yano S, Chattopadhyay $\mathrm{N}$ and Brown EM: Extracellular calcium-sensing receptor transactivates the epidermal growth factor receptor by a triple-membrane-spanning signaling mechanism. Biochem Biophys Res Commun 320: 455-460, 2004 